\title{
Pulmonary arterial distensibility - 2D phase contrast vs $2 \mathrm{D}$ bSSFP
}

\author{
Alejandro Roldán-Alzate ${ }^{1 *}$, Oliver Wieben ${ }^{1,2}$, Alex Frydrychowicz $^{1}$, Naomi C Chesler ${ }^{3}$, Christopher Francois $^{1}$ \\ From 15th Annual SCMR Scientific Sessions \\ Orlando, FL, USA. 2-5 February 2012
}

\begin{abstract}
Summary
Main pulmonary artery distensibility is a strong predictor of mortality in patients with pulmonary arterial hypertension. This study shows that either 2D PC or 2D bSSFP can be used to reliably non-invasively assess it by measuring MPA relative area change.
\end{abstract}

\section{Background}

The pulmonary circulation is a highly compliant system that generates little resistance to blood flow. However, in the presence of pulmonary arterial hypertension (PAH), blood pressure and vascular resistance in the pulmonary circulation are elevated. This leads to distension and stiffening of the main pulmonary artery (MPA) and vessel wall remodeling, which in itself may influence stiffness [1]. Proximal arterial distensibility is a parameter that depends mainly on the anatomy (geometry) of the vessel and can be estimated noninvasively with cross-sectional imaging techniques. In particular, the relative area change (RAC) of the MPA, which is inversely proportional to arterial stiffness, is a strong predictor of mortality in patients with PAH [2]. The purpose of this study was to compare two non-invasive magnetic resonance imaging (MRI) methods for quantification of MPA distensibility using an acute PAH dog model.

\section{Methods}

Six adult female beagles were anesthetized with isoflurane. MRI was performed prior to and following injection of micro-beads into the right atrium and ventricle to induce PAH, resulting 12 comparison studies. The presence of $\mathrm{PAH}$ was confirmed by right heart catheterization (RHC). All MR images were acquired on a $3 \mathrm{~T}$ scanner (MR750, GE Healthcare, Waukesha, WI). Double-oblique images perpendicular to the direction of the

${ }^{1}$ Radiology, University of Wisconsin, Madison, WI, USA

Full list of author information is available at the end of the article flow in the MPA were obtained using ECG-triggered 2D CINE balanced steady-state free precession (bSSFP) and through-plane velocity-encoded 2D phase contrast (PC) at the same level [3]. PC and bSSFP images were segmented using dedicated cardiovascular analysis software (CV-Flow and and MASS-Analysis, respectively, Medis, Leiden, NL). Maximum and minimum MPA areas $\left(\mathrm{A}_{\max }\right.$ and $A_{\min }$, respectively) were used to calculate $\mathrm{RAC}=$ $\left(A_{\max }-A_{\min }\right) / A_{\max }$. Bland-Altman analysis was used to study the differences between PC and bSSFP to calculate $A_{\max }, A_{\min }$, and RAC. Student t-test was used to evaluate statistical significance of the differences between techniques in all three paramenters.

\section{Results}

The mean values for $\mathrm{RAC}, \mathrm{A}_{\max }$ and $\mathrm{A}_{\min }$ were 36.38 $\pm 7.86 \%, 241.98 \pm 71.62 \mathrm{~cm}^{2}$, and $157.06 \pm 58.51 \mathrm{~cm}^{2}$ for $\mathrm{PC}$, respectively and $31.14 \pm 6.95 \%, 220.88 \pm 62.76 \mathrm{~cm}^{2}$, and $155.34 \pm 58.38 \mathrm{~cm}^{2}$ for bSSFP, respectively $(\mathrm{p}=0.10,0.45$, and 0.94, respectively). The mean differences for RAC, $\mathrm{A}_{\max }$ and $\mathrm{A}_{\min }$ were $-5.23 \pm 8.44 \%,-21.10 \pm 40.67 \mathrm{~cm}^{2}$, $-1.72 \pm 40.89 \mathrm{~cm}^{2}$, respectively.

\section{Conclusions}

No statistically significant difference is present between PC and bSSFP for measuring RAC, $\mathrm{A}_{\max }$ or $\mathrm{A}_{\text {min }}$. The differences between the two methods for measuring these parameters are small suggesting that either technique (PC or bSSFP) can be used to reliably non-invasively measure MPA distensibility. A benefit of using PC for measuring RAC is that it can also be used to quantify blood flow.

\section{Funding}

University of Wisconsin - Madison, Department of Radiology, Research and Development fund. 

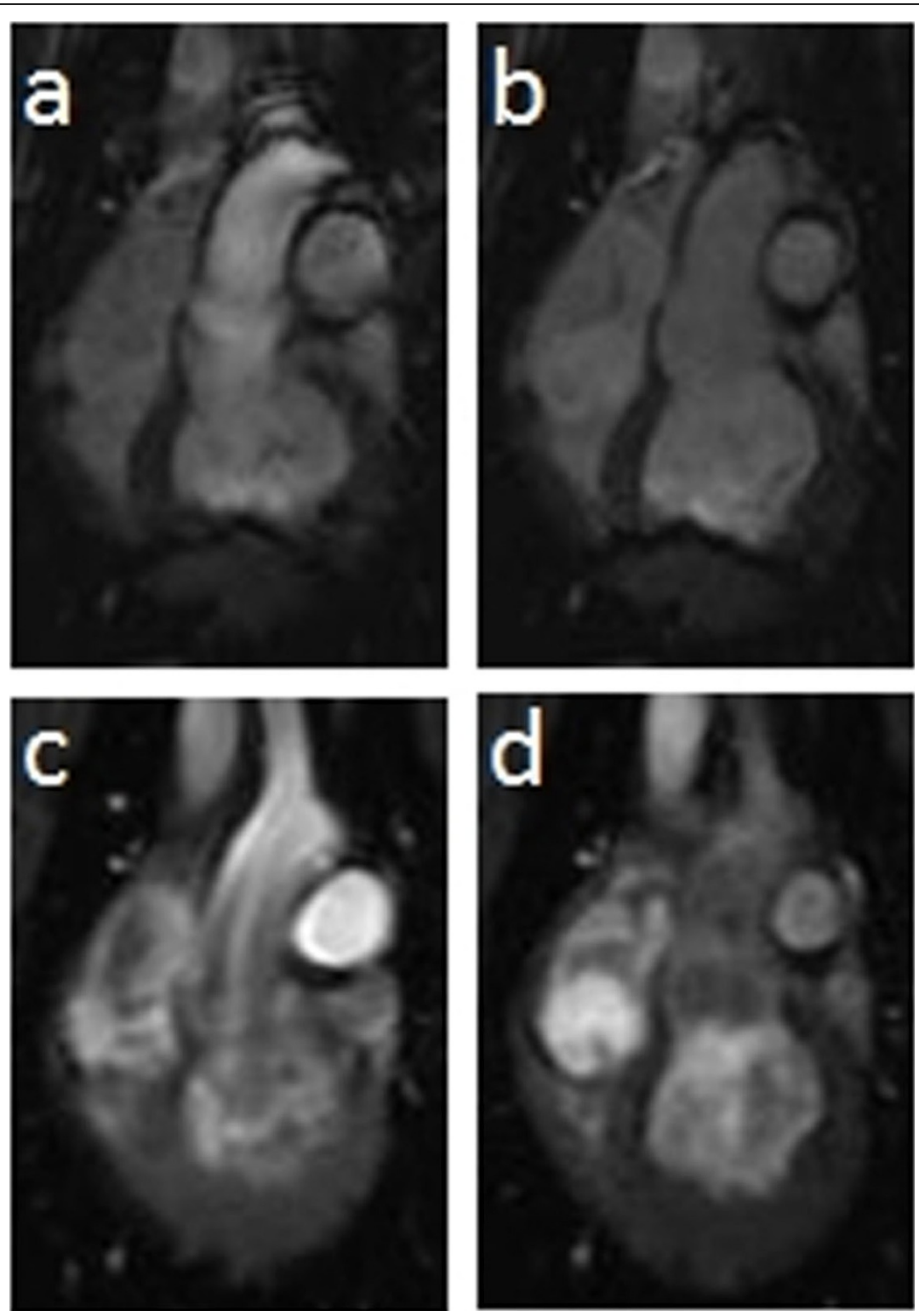

Figure $12 D$ bSSFP images (a. $A_{\max }$ and b. $A_{\min }$ ) and 2DPC images (c. $A_{\max }$ and d. $A_{\min }$ ).

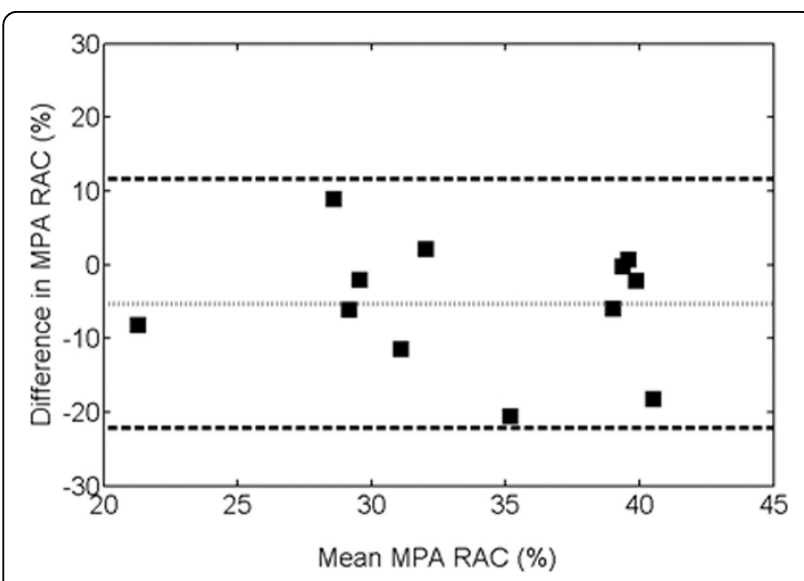

Figure 2 Bland-Altman plot for comparison of MPA RAC measured with $2 \mathrm{D} P \mathrm{PC}$ and $2 \mathrm{D}$ bSSFP.

\section{Author details}

${ }^{1}$ Radiology, University of Wisconsin, Madison, WI, USA. ${ }^{2}$ Medical Physics,

University of Wisconsin, Madison, WI, USA. ${ }^{3}$ Biomedical Engineering, University of Wisconsin, Madison, WI, USA.

Published: 1 February 2012

\section{References}

1. Boerrigter B: Chest. 2010

2. Gan C: Chest. 2007.

3. Herment A: MRM. 2011.

doi:10.1186/1532-429X-14-S1-P85

Cite this article as: Roldán-Alzate et al:: Pulmonary arterial distensibility 2D phase contrast vs 2D bSSFP. Journal of Cardiovascular Magnetic

Resonance 2012 14(Suppl 1):P85. 